\title{
A statistical method for driving cycle construction based on path geometry
}

\author{
Mingyue Ma \\ School of Transportation Science and Engineering \\ Beijing University of Aeronautics and Astronautics \\ Beijing, China \\ mmy@ae.buaa.edu.cn
}

\author{
Ferit Küçükay \\ Institute of Automotive Engineering \\ TU Braunschweig \\ Braunschweig, Germany \\ f.kuecuekay@tu-braunschweig.de
}

\author{
Benedikt Weiler \\ Institute of Automotive Engineering \\ TU Braunschweig \\ Braunschweig, Germany \\ b.weiler@tu-braunschweig.de
}

\author{
Xiangyang $\mathrm{Xu}$ \\ School of Transportation Science and Engineering \\ Beijing University of Aeronautics and Astronautics \\ Beijing, China \\ xxy@buaa.edu.cn
}

\begin{abstract}
This paper developed a method for constructing a driving cycle for vehicle simulation and analysis reflecting the driver behavior in real traffic condition based on path geometry. Markov Chain approach was employed to construct a model to generate the driving cycle comprised of three cycle profiles i.e., speed profile, gradient profile and curvature profile. The driving cycle is derived from extensive testing data under the real driving condition through a series of statistical analysis.
\end{abstract}

Index Terms-Driving cycle, Markov Chain, Statistics.

\section{INTRODUCTION}

There are two main methods to generate driving cycle used for vehicle testing and vehicle simulation. One is derived from the standard driving cycles which are widely used for statutory vehicle emission test and vehicle fuel economy estimation. However, the standard driving cycle is not able to satisfy the requirement of various driving condition under the real traffic environment in the distinct country and area. Another method is to construct the driving cycle based on the statistical analysis of raw vehicle testing data. Compared to the first method, the second method can construct the driving cycle reflecting more characteristic of the driving condition of the corresponding area. Therefore, a number of methods have been proposed to construct a driving cycle with the corresponding vehicle test data. Driving cycle for Hong Kong was presented by summarizing the statistics of the sample that was derived from on-road testing data [1]. Then for improving the synthesized driving cycle of Hong Kong the performance value and speedacceleration probability distribution were introduced to generate the driving cycle [2]. A reference cycle was used as a training set to synthesize the driving cycle on the basis of the method of Markov Chain [3]. But all of methods have not considered the path geometry which has a significant effect to driving pattern. For example, the performance of the vehicle on the flat path cannot be as same as the one on the curve path or in the hill area. A gradient-sensitive driving cycle for vehicles in military areas was developed and the effect of the terrain was considered firstly [4]. In this work, a new method considering the effect of path geometry including the gradient and the curvature based on Markov Chain approach used to generate a driving cycle was developed.

\section{DATA COLLECTION}

The raw data used for generating the driving cycle in this paper was derived from the long distance truck testing which is conducted by the Institute of Automotive Engineering of TU Braunschweig. The selected road type of these tests consists of the urban, country and highway. This testing data was collected on six hundred and twenty-five trips. The minimum distance of one trip is $6.5 \mathrm{~km}$, the maximum distance of one trip is 465.3 $\mathrm{km}$ and the total test distance is more than $150,000 \mathrm{~km}$. The figure 1 shows a part of the testing route on Google map recorded by GPS. The data of each trip comprises a number of variables used to represent the movement of the engine, the movement of the vehicle, the operation of the driver, etc.

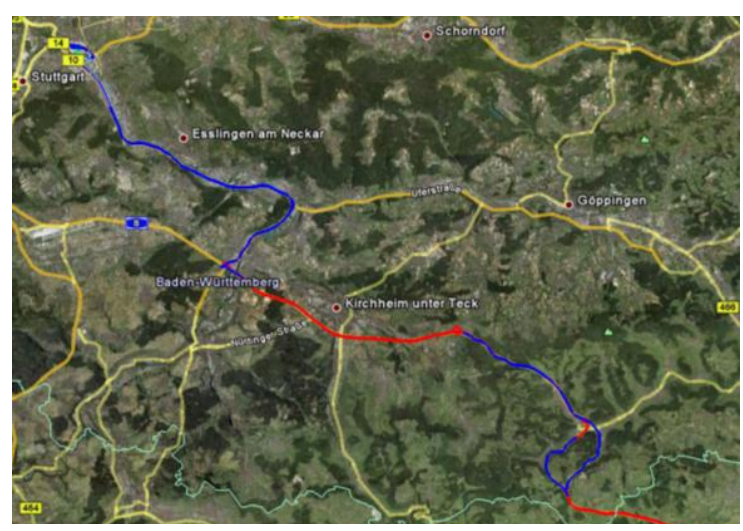

Fig. 1. A part of the testing route. 
The variables involved in this study are the speed, gradient and curvature, of which the curvature cannot be measured indirectly. Nevertheless, the curvature is able to be calculated to make use of the wheel speed difference between the left wheel and the right wheel on the curve path. The curvature is defined by

$$
1 / R=2 / w *\left(v_{-} L-v_{-} R\right) /\left(v_{-} L+v_{-} R\right) .
$$

Where $R$ is the radius of the vehicle, $w$ is the wheel width, $v_{-} L$ is the left wheel speed and $v_{-} R$ is the right wheel speed.

\section{DATA ANALYSIS}

The process of the measurement data is carried out to simplify and quantize the data before the statistical analysis. The method used to processing data in this work is proposed by the Institute of Automotive Engineering of TU Braunschweig []. After the process the raw data with waves can be transformed into the smooth profile which is just able to represent those distinct transitions of the data. The newly obtained profile is called as the orientation profile. Thus, the orientation speed profile, the orientation gradient profile and the orientation curvature profile can be obtained after this process. For example, we can see the comparison of the original speed profile (OSP) and the orientation speed profile in Figure 1.

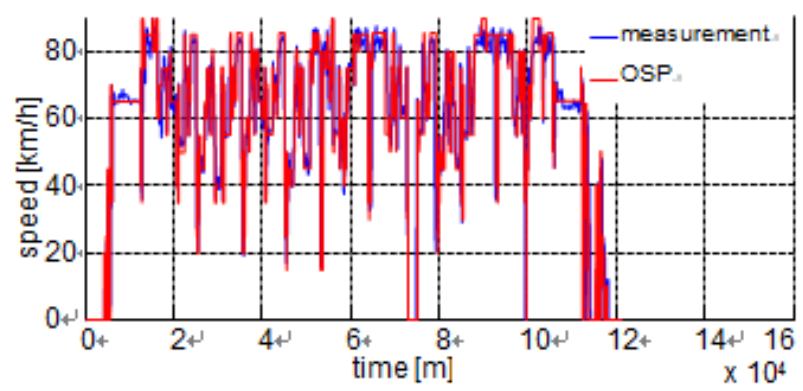

Fig. 2. The comparison of the orientation profile and the raw data.

Based on existing models of data processing as for velocity and gradient, the orientation speed profile and the orientation gradient profile (OGP) can be obtained, respectively. The comparison of the orientation profile and the raw data can be shown in (figure 1). The effectiveness of the orientation profile is that to classify the data of the variable to comply with some constraints. The interval of the speed for OSP is set to $5 \mathrm{~km} / \mathrm{h}$ and the one of the gradient for OGP is set to $0.5 \%$. Thus the value of the speed corresponding to class 1 in the OSP is 0 $\mathrm{km} / \mathrm{h}$, to class 2 is $5 \mathrm{~km} / \mathrm{h}$ and so on. Similarly the value of the gradient corresponding to class 1 in the OGP is $-30 \%$, to class 2 is $-29.5 \%$ and so on, of which the value corresponding to class 31 is $0 \%$ and represents the even road without slopes.

When the vehicle begins to be driven on the curve road or driven off the curve road, the curvature of the pathway of the vehicle doesn't change suddenly in the real traffic condition. If not, the driver and the passenger can feel very uncomfortable. Therefore the curvature changes step by step at the beginning and end of the curve. The clothoid curve is always used in the simulation of the curve pathway. There are a number of researches of the clothoid curve applied to represent the curve pathway of the vehicle and the results have been tested by researchers [5-6]. As for the measurement of the curvature in this work, a clothoid curve is used to simplify the curvature profile. Based on the approximate curvature profile the orientation curvature profile (OCP) can be obtained [7].

The measurement of the variable mentioned above will be quantized and then some specific points which represent the jumping of the corresponding variable in the process. Figure 3 shows a part of orientation profiles of speed, gradient, curvature respectively. And then, we can see in the figure there are a number of points marked by small cycle in every orientation profile at which the values of the variables are changed. These points are called as jumping points. The moment and parameters of the jumping point are recorded and then a database used to save the data is able to be constructed. Every 2 adjacent jumping points can be seen as one pair. Every jumping point carries information including the occurring moment, as well as the value of speed, gradient and curvature. There is a connection between one pair of the jumping points. As for one pair of jumping points, the first point appears firstly and then the second point appears. There always exists one constant probability to estimate the occurrence between a pair of jumping points. According to these points the whole process can be divided into many individual segments. The values of speed, gradient and curvature keep constant in the same segment. There is a need to present a definition which is used to represent the current state and the next state of Markov Chain. The variables of speed, gradient and curvature are stored in the same segment as a vector. This vector is defined as the state of Markov Chain and then called as maneuver vector of this segment. A complete profile consists of a series of ordered maneuver vectors. The maneuver vector could be same though it belongs to different segments.

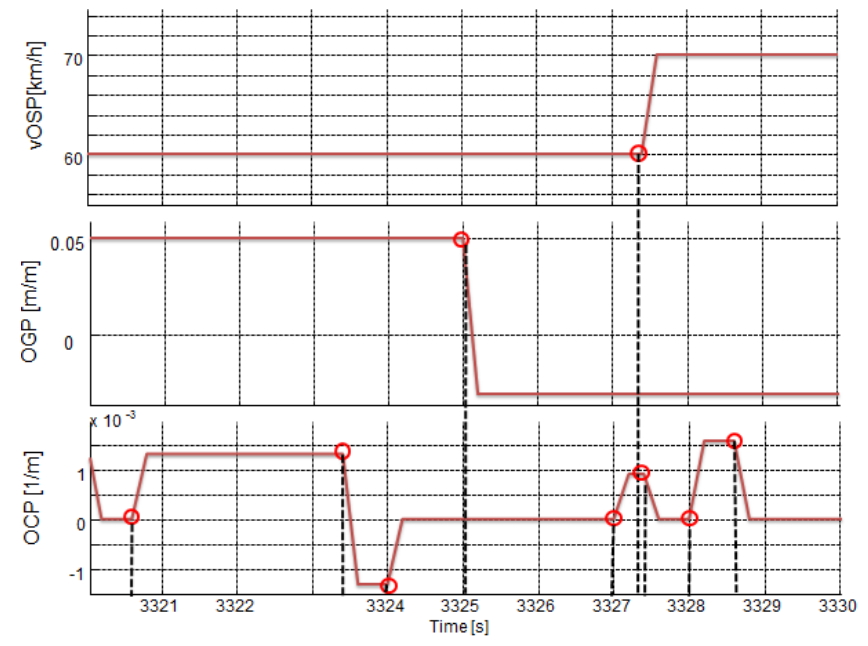

Fig. 3. Collection of the jumping point 


\section{THE METHOD FOR GENERATING DRIVING CYCLE}

The method used to generate a multi-parameter driving cycle is an iterative Markov Chain approach. The most important part for making use of Markov Chain approach is to build a state transition matrix to characterize the probability of the current state changed to the next state. The formula used to calculate the probability of the transition of maneuvers is

$$
p(i, j)=n \_(i, j) / n \_i \text {. }
$$

Where $n \_(i, j)$ is the number of occurrences of the transition from maneuver $i$ to maneuver $j . n_{-} i$ is the total number of occurrences of the maneuver $i$.

The first step of this method is the construction of the transition probability matrix. It can be used to get the next maneuver randomly in the following step if the current maneuver is known. The operation to build the transition matrix is as following: firstly summarize all the transition between two different maneuvers, secondly count the number of the occurrences of every transition, and then count the number of the occurrences of every maneuver which is as a starting maneuver in the transition. At last compute the transition probability of each state transition. Figure 4 shows the probability distribution obtained from a part of the transition matrix. X Coordinate represents the information of the first point, and y Coordinate represents the information of the second point. The different color means frequency of occurrence of the transition of maneuvers.

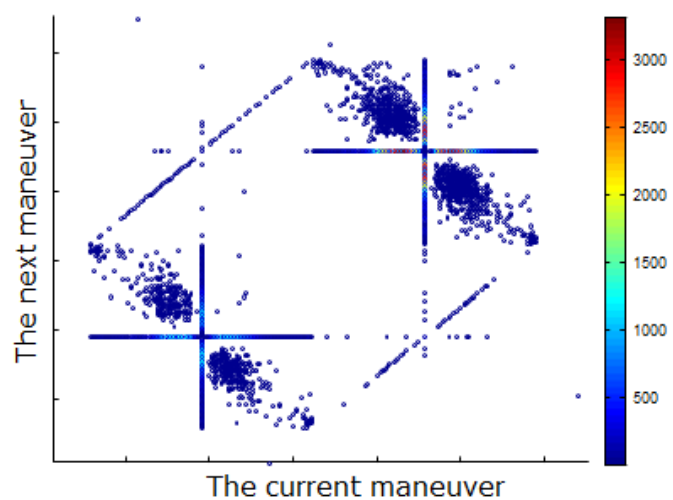

Fig. 4. The probability distribution of transition matrix of maneuvers

For obtaining a more accurate speed profile, gradient profile and curvature profile, a velocity constrain is necessary to introduced in this method. This velocity constrain represents the Upper and lower limits of the driving distance when the velocity is changed. Figure 5 shows the statistic results of driving distance from the moment when velocity begins to change to the moment before velocity changes again. As for each condition, select the maximum and minimum value of the distance as the velocity constrain.

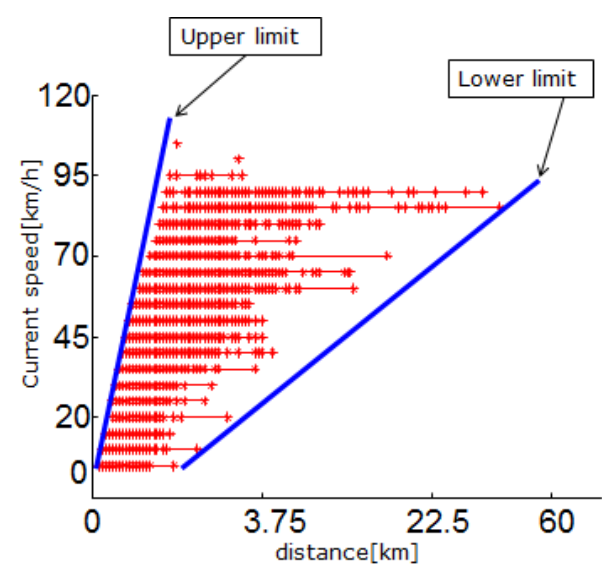

Fig. 5. The distance constraints for the current speed $=0$.

The process of generation new speed profile is as following: Firstly select the initial value, and then according to the transition probability distribution of this state select the next step randomly. Determine whether the constraint of the velocity is satisfied. If so, continue to select the next state. If not, the selected state will be deleted. This process will be iterated until the initial distance is achieved.

\section{CONCLUSION}

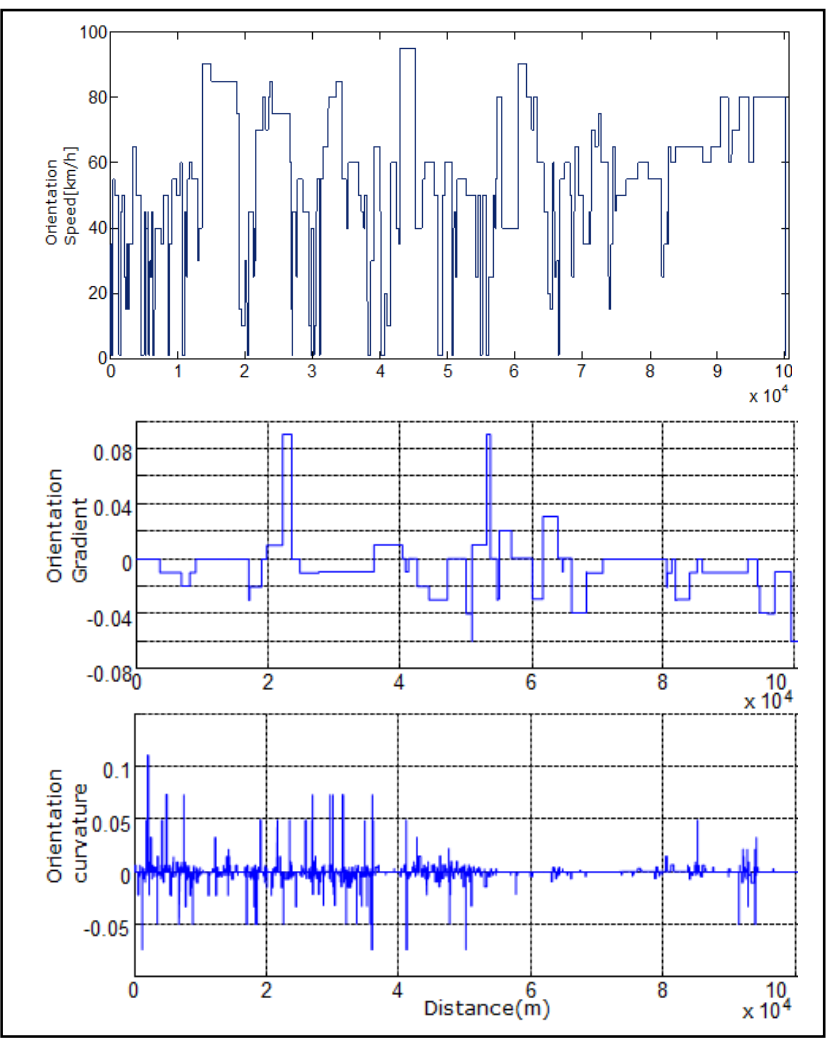

Fig. 6. A driving cycle including gradient and curvature. 
With this approach, the orientation speed profile, orientation gradient profile and orientation curvature profile can be generated. To insure the qaulity of the synthesied speed, gradient and curvature profiles the statistics of each synthesied profile must be compared to that of the raw data. The composite profile within $5 \%$ difference from the raw data can be accepted. We can see one group of the acceptable results in the figure 6 . These results can be used together when the vehicle simulation need to consider the speed, gradient and curvature at the same time. Furthermore as for the different need the corresponding parameter profile can be applyed independently.

\section{ACKNOWLEDGMENT}

The work described in this paper was supported by the Institute of Automotive Engineering of TU Braunschweig. The testing data used in this paper was derived from the database of the Institute of Automotive Engineering of TU Braunschweig.

\section{REFERENCES}

[1] H.Y. Tong, W.T. Hung and C.S. Cheung, "Development of a driving cycle for Hong Kong," Atmospheric Environment, vol. 33, pp. 2323-2335, 1999.
[2] W. T. Hung, H. Y. Tong, C. P. Lee, K. Ha and L. Y. Pao, "Development of a practical driving cycle construction method: A case study in Hong Kong," Transportation Research Part D: Transport and Environment, vol. 12, pp. 115-128, 2007.

[3] A. Ivanco, A. Charlet, Y. Chamaillard and P. Higelin, "Energy Management Strategies for Hybrid-Pneumatic Engine Studied on an Markov Chain Type Generated Driving Cycle," SAE, 2009-01-0145.

[4] D. S. Han, N. W. Choi, S. L. Cho, J. S. Yang, K. S. Kim, W. S. Yoo and C. H. Jeon, "Characterization of driving patterns and development of a driving cycle in a military area," Transportation Research Part D: Transport and Environment, vol. 17, pp. 519-524, 2012.

[5] D. Khosla, "Accurate estimation of forward path geometry using two-clothoid road model," Intelligent Vehicle Symposium, 2002. IEEE, vol. 1, pp. 154 - 159, June 2002.

[6] H. Loose and U. Franke, "B-Spline-Based Road Model for 3D Lane Recognition," 13th International IEEE Annual Conference on Intelligent Transportation Systems, Madeira Island, Portugal, September 19-22, 2010.

[7] U. Hintze, "Erweiterung eines statistischen Fahrumgebungsmodells zur Berücksichtigung von Geländetopologien," unpublished. 